\title{
CLINICOPATHOLOGICAL STUDY OF FUNGAL BURSITIS
}

\author{
Shima Kannadas ${ }^{1}$, Thundiparambil Raghavan Nisha
}

${ }^{1}$ Senior Resident, Department of Pathology, Government Medical College, Kozhikode.

${ }^{2}$ Assistant Professor, Department of Pathology, Government Medical College, Kozhikode.

\section{ABSTRACT}

\section{BACKGROUND}

Bursitis is described as inflammation of a bursa. Bursitis may result from several causes like1,2 direct injury or trauma, prolonged pressure, overuse or strenuous activity, crystal-induced arthropathy, inflammatory arthritis and infection (septic bursitis). The majority of cases of septic bursitis are due to bacterial pathogens. More recently, reports have appeared in the literature of chronic infectious bursitis caused by fungi and occurring in both immunocompromised and healthy immunocompetent individuals. ${ }^{3}$

\section{MATERIALS AND METHODS}

This is a descriptive study done from 2009 to 2015 conducted in the Department of Pathology, Government Medical College, Kozhikode. Study sample included retrospective material from 2009 to 2014 and prospective material from 2014 to 2015 . The risk factors and clinical findings were assessed. The blocks of all specimens were cut and stained with $\mathrm{H} \& \mathrm{E}$ and fungal stains.

\section{RESULTS}

Out of the 45 cases of bursitis, 10 cases were diagnosed as fungal bursitis. 5 out of 10 cases were Masson-Fontana positive and diagnosed as Phaeohyphomycosis. 90\% sustained injuries at the site of fungal bursitis. $70 \%$ of the cases with fungal bursitis had diabetes. Fungal bursitis was found to be more prevalent in the olecranon bursa (60\%).

\section{CONCLUSION}

This study suggests that fungal bursitis is not a rare entity. The prevalence of fungal bursitis is more in olecranon bursa and in diabetic patients. The most common diagnosis was phaeohyphomycosis.

\section{KEYWORDS}

Fungal Bursitis, Phaeohyphomycosis, Olecranon Bursitis.

HOW TO CITE THIS ARTICLE: Kannadas S, Nisha TR. Clinicopathological study of fungal bursitis. J. Evolution Med. Dent. Sci. 2017;6(47):3629-3632, DOI: 10.14260/Jemds/2017/782

\section{BACKGROUND}

Bursae are sac like structures between skin and bone or between tendons, ligaments, and bone lined by synovial tissue. Common sites for bursitis to develop are olecranon, psoas tendon, greater trochanter, iliotibial band, prepatellar, infrapatellar and retrocalcaneal. Septic bursitis is a commonly encountered condition. Because of the location of the olecranon and prepatellar bursae, they are the most frequently affected sites. The majority of cases of septic bursitis are due to bacterial pathogens, particularly Grampositive organisms (Staphylococcus aureus and Streptococcal species). In chronic bursitis, unusual organisms should be considered, including Mycobacterium tuberculosis, NTM, Brucella spp., fungi, or molds. Fungal bursitis though rare, is important clinically because the cases which do not respond to the usual treatment protocol may harbour an underlying infectious aetiology.

Clinical features include erythema, swelling, and pain with a point of maximal central tenderness. Fever, cellulitis, and other signs of skin compromise may be noted.

Financial or Other, Competing Interest: None.

Submission 30-04-2017, Peer Review 30-05-2017,

Acceptance 05-06-2017, Published 12-06-2017.

Corresponding Author:

Shima Kannadas,

Aksharam, Kakkodi (P. O.),

Calicut, Kerala, India.

E-mail:drkshima@gmail.com

DOI: $10.14260 /$ jemds $/ 2017 / 782$
For diagnosis of fungal bursitis, bursal fluid aspiration, synovial fluid analysis and culture are essential. Imaging with US and CT can help in the diagnosis and localisation of pathology.

Histopathological examination of the excised bursa may also be helpful. H\&E staining can be used to know the presence of features of fungal infection like necrosis, histiocytic collections, inflammatory infiltrates, etc. It can also help in the demonstration of some of the fungi. Other special stains done routinely include Gomori methenamine silver (GMS), Periodic acid-Schiff (PAS), Gridley's method, Mucicarmine and Masson-Fontana (for pigmented fungi). In the present study, the stains used include $\mathrm{H} \& \mathrm{E}, \mathrm{GMS}$, PAS and Masson-Fontana.

Very few studies were reported in literature regarding the clinic morphological features of fungal bursitis. This study aimed to describe the clinical features and morphology of fungal bursitis diagnosed in our centre and to delineate the risk factors for the disease.

\section{MATERIALS AND METHODS}

A descriptive study was conducted in the department of Pathology, Government Medical College, Calicut. Study sample included all cases of bursitis diagnosed during the period 2009 to 2015 . The clinical history was collected from the case sheets and operation notes as well as Pathology requisition forms. Relevant clinical findings like history of diabetes, injury sustained, site and nature of swelling, drug history, history of immunodeficiency, etc. were noted. The 
blocks of all specimens were cut at $5 \mathrm{~mm}$ and stained with H\&E, Gomori's methenamine silver, PAS and MassonFontana.

\section{RESULTS}

Histopathological examination of total of 45 cases were studied. Mean age of the patients from whom the samples received is 49 years, maximum being 81 and minimum 8 . Out of the 45 cases studied, 9 cases sustained injuries at the site of bursitis. No history of intake of immunosuppressive drugs including steroids was present in the study group.

$15 \%$ of the cases were hypertensive and $33 \%$ were diabetic. The common sites of bursitis in our study group were olecranon and patella both of which constitute 34 out of the 45 cases (75\%) [Figure 1].

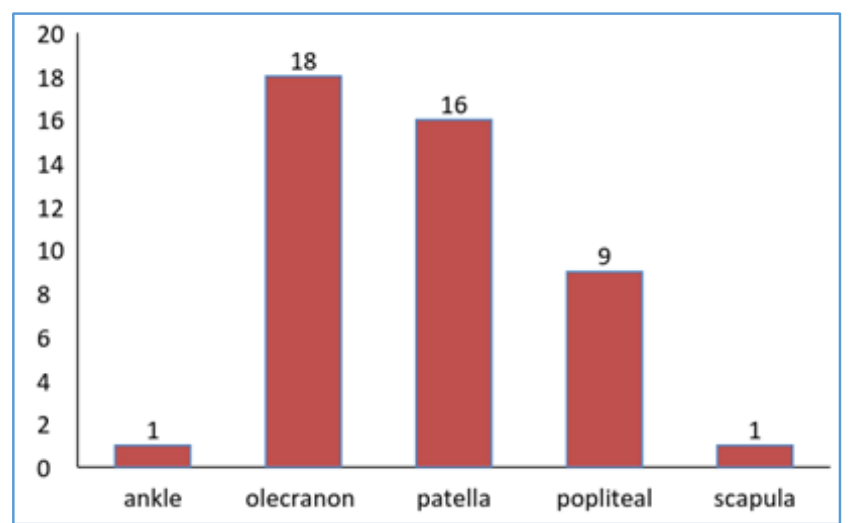

Figure 1. Frequency Distribution of Bursitis in Various Sites

10 out of 45 cases showed evidence of fungal infection in H\&E like necrosis, histiocytic collections and inflammatory infiltrates. 7 out of the 10 cases showed areas of necrosis with mixed inflammatory infiltrates composed of lymphocytes, neutrophils and eosinophils.

2 cases showed palisading granulomas with abundant epithelioid macrophages and multinucleate giant cells (Figure 2).

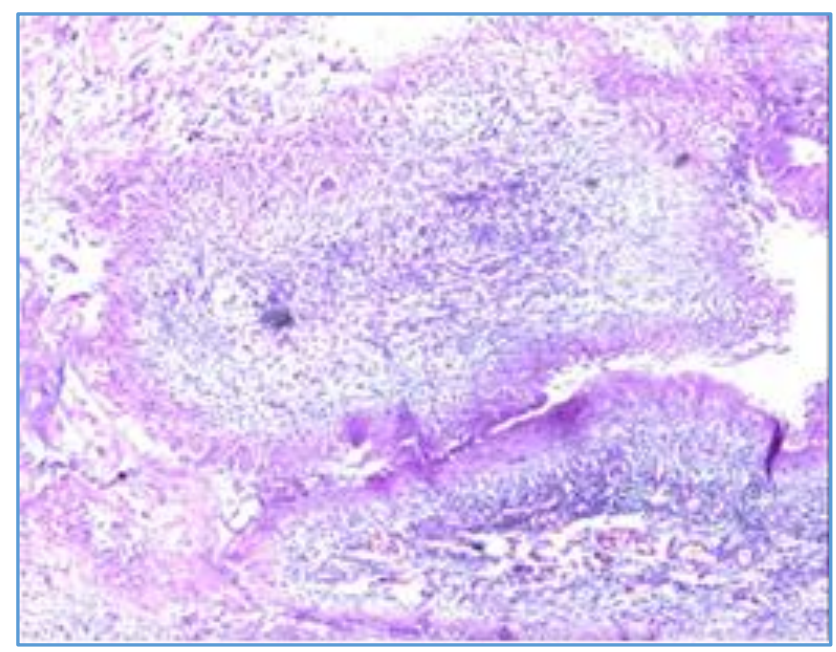

Figure 2. H \& E showing Palisaded Granuloma

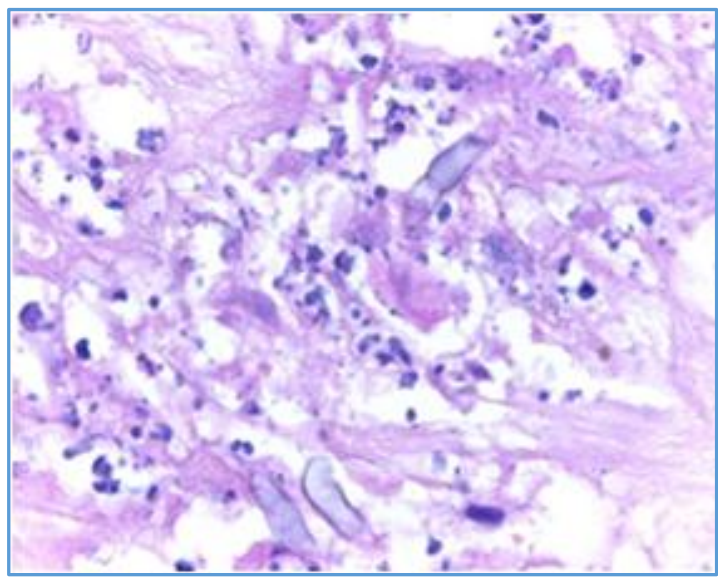

Figure 3. Case of Fungal Bursitis showing Areas of Necrosis with Fungal Hyphae (100x)

All the 10 cases showed positivity with GMS. MassonFontana was positive in 5 of these 10 cases indicating phaeohyphomycosis. Of the 5 cases of phaeohyphomycosis, 5/5 cases showed necrosis and 2 showed palisading granuloma.

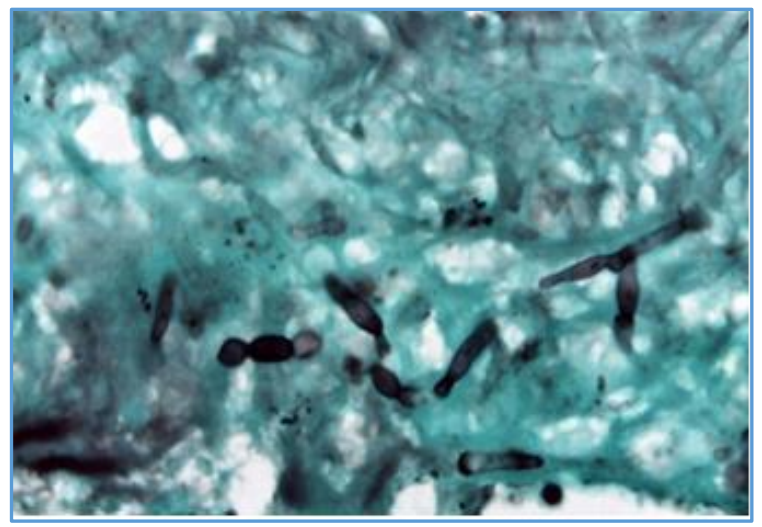

Figure 4. GMS Stain showing Septate Fungal Hyphae [40x]

One case of phaeohyphomycosis showed areas of necrosis with foamy macrophages, multinucleate giant cells and epithelioid cells. Fungal hyphae could be detected in H\&E itself. GMS stain clearly outlined the fungi having septate hyphae and Masson-Fontana showed blackish pigments suggestive of phaeohyphomycosis (Figure 5).

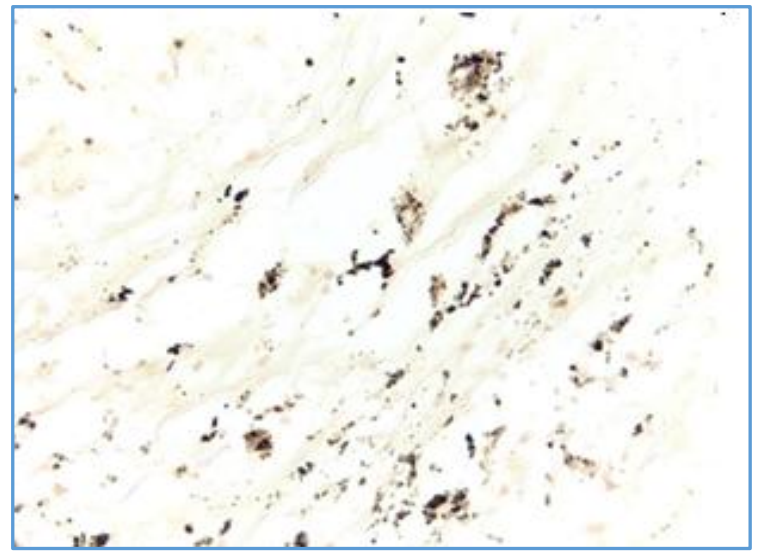

Figure 5. Fungal Pigments Stained with Masson-Fontana [10x] 
Of the cases diagnosed as fungal bursitis, 7 were male and the rest were female. All cases belong to age group of $>40$ years. 9 cases sustained injuries at the site of bursitis. 7 patients were diabetics, 1 patient had hypertension and 1 out of 10 cases was alcoholic. 6 out of 10 cases were firm swellings, and the rest were cystic associated with pain. In the present study, the most common site of fungal bursitis was olecranon ( $6 / 10$ cases).

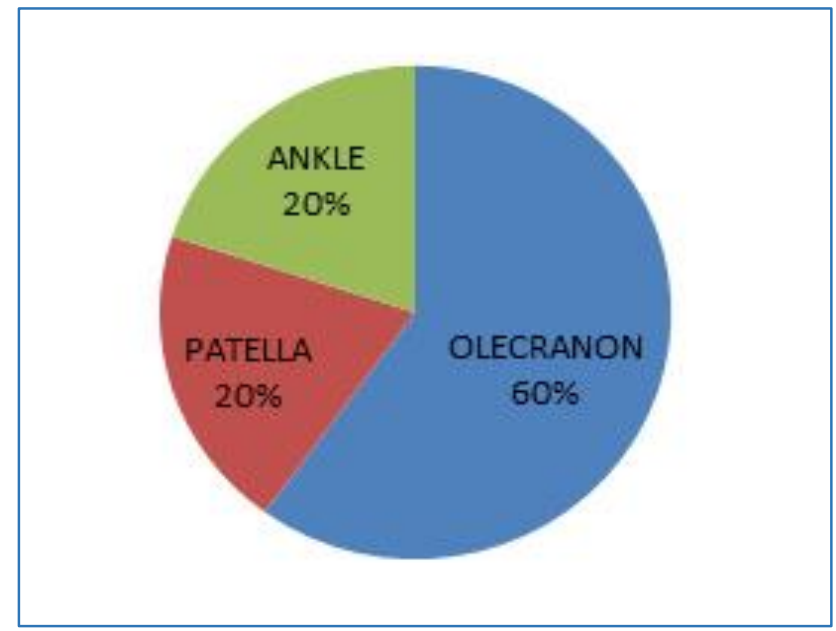

Figure 6. Distribution of Fungal Bursitis

\section{DISCUSSION}

The most common causes of bursitis are repetitive motions, trauma, infection, and pre-existing rheumatoid conditions. Bursae close to the surface of the skin are most likely to get infected. People with diabetes, alcoholism, certain kidney conditions, those with suppressed immune systems such as from cortisone medications (steroid treatments), and those with wounds to the skin over a bursa are at higher risk for fungal bursitis.

Most fungi can be identified with the standard haematoxylin and eosin (H\&E) stain. The identification of fungi in biopsy samples is enhanced by the selective application of widely available histochemical stains. The commonly used fungal stains are: Gomori methenamine silver (GMS), Periodic acid-Schiff (PAS), Gridley's method and Mucicarmine. The Gomori methenamine silver (GMS) and Gridley stains are the preferred methods for demonstrating fungi. Because certain fungi demonstrated by GMS do not consistently stain well with periodic acid-Schiff (PAS), the latter should be reserved as a secondary approach, but it can at times enhance morphologic detail.

Tissue sampling is fundamentally important in the diagnosis. All excised tissues should be considered as potentially infective. This approach fosters due diligence with respect to the possibility of contamination, as well as thoughtful concern as to how the tissues will be handled to optimise the chances of establishing an accurate diagnosis. Samples of excised tissues should be harvested by sterile technique. What must be avoided is thoughtlessly placing a biopsy specimen directly into formalin fixative without first considering a diagnosis of infection.

In the present study, out of 45 cases, 10 cases were diagnosed as fungal bursitis. Histopathology of 7 out of 10 cases showed large areas of necrosis with mixed inflammatory infiltrate. Histiocytic infiltrate, predominantly foamy macrophages, was seen in one of the cases. No significant inflammation or necrosis was observed in 1 case. Granuloma with multinucleate giant cells and palisading necrosis suggestive of fungal bursitis was found in 2 out of the 10 cases of bursitis.

All these cases show positivity with GMS in the form of fungal hyphae and spores and were negative for PAS. This shows that GMS staining is more useful in the detection of fungal bursitis. 5 were Masson-Fontana positive indicating phaeohyphomycosis. Of the 5 cases of phaeohyphomycosis, $5 / 5$ cases showed necrosis and 2 showed palisading granuloma.

All cases of fungal bursitis belong to age group of $>40$ years, maximum age being 81 and minimum 44 . Thus, the prevalence of fungal bursitis is found to be more in older age group. 9 out of 10 cases sustained injuries at the site of fungal bursitis, this reinforces the fact that trauma favours the transcutaneous transit of fungi to the bursa.1,2,4 One study of septic olecranon bursitis found that one-third of patients had at least one comorbid illness. ${ }^{5}$ It is unclear whether immunosuppressed states such as HIV/AIDS increase the risk of septic bursitis. ${ }^{6,7}$

The majority of reported cases of septic bursitis have been in men.5,8,9,10 Trauma to the skin and bursa may be occupational, due to recreational activities, or related to concomitant diseases. In our study, 7 cases were male and 3 were female.

Although the prevalence of bursitis, in total, was equal in olecranon as well as patellar bursa; fungal bursitis was found to be more prevalent in the olecranon bursa. This was mentioned in one study ${ }^{11,12}$ that due to its superficial location, swelling of the olecranon bursa is easy to detect by both the patient and the examiner. Other less common sites of involvement in fungal bursitis were patella and ankle (retrocalcaneal). Direct inoculation of deep bursa is uncommon, but iatrogenic infection resulting from bursal injections of glucocorticoids (e.g., subacromial, iliopsoas, or trochanteric bursae) may rarely occur. ${ }^{13}$. In the absence of a known inoculation, infection of deep bursae are presumed to be due to haematogenous seeding or spread from an adjacent septic site such as contiguous septic arthritis. ${ }^{14}$

Almost all cases of paediatric septic bursitis occur after acute trauma, with or without skin abrasion, rather than after repetitive occupational trauma. This mimics the paediatric model of acute osteomyelitis, which is similarly associated with minor trauma and which is haematogenous in origin. ${ }^{15}$

In the study population, prevalence of diabetes was 33\%. 7 out of the 10 cases with fungal bursitis had diabetes. This signifies the role of diabetes mellitus as a risk factor. Out of the 8 cases which were studied prospectively, all were negative for fungal culture as well as fungal stains. Culture was not done in any of the retrospective cases.

No history of intake of immunosuppressive drugs was obtained in any of the 10 cases of fungal bursitis. This reestablishes older studies which claim that fungal bursitis can occur in immunocompetent individuals too. ${ }^{3}$

\section{CONCLUSION}

This study suggests that possibility of fungal infection should be considered while dealing with all cases of bursitis. In our study, prevalence of fungal bursitis was found to be more in diabetics and those prone to injuries, and majority of the 
cases belong to phaeohyphomycosis. Larger cohorts need to be studied to ascertain the accurate epidemiological profile of fungal bursitis.

\section{REFERENCES}

[1] Ho G, Tice AD, Kaplan SR. Septic bursitis in the prepatellar and olecranon bursae: an analysis of 25 cases. Ann Intern Med 1978;89(1):21-7.

[2] Canoso JJ, Yood RA. Reaction of superficial bursae in response to specific disease stimuli. Arthritis Rheum 1979;22(12):1361-4.

[3] Gertner E. Chronic septic bursitis caused by dematiaceous fungi. Am J Orthop 2007;36(1):E10.

[4] Valeriano-Marcet J, Carter JD, Vasey FB. Soft tissue disease. Rheum Dis Clin North Am 2003;29(1):77-88.

[5] Laupland KB, Davies HD, Calgary Home Parenteral Therapy Program Study Group. Olecranon septic bursitis managed in an ambulatory setting. The Calgary home parenteral therapy program study group. Clin Invest Med 2001;24(4):171-8.

[6] Zimmermann B 3rd, Mikolich DJ, Ho G. Septic bursitis. Semin Arthritis Rheum 1995;24(6):391-410.

[7] Söderquist B, Hedström SA. Predisposing factors, bacteriology and antibiotic therapy in 35 cases of septic bursitis. Scand J Infect Dis 1986;18(4):305-11.
[8] Cea-Pereiro JC, Garcia-Meijide J, Mera-Varela A, et al. A comparison between septic bursitis caused by Staphylococcus aureus and those caused by other organisms. Clin Rheumatol 2001;20(1):10-4.

[9] García-Porrúa C, González-Gay MA, Ibañez D, et al. The clinical spectrum of severe septic bursitis in northwestern Spain: a 10 year study. J Rheumatol 1999;26(3):663-7.

[10] Stell IM, Gransden WR. Simple tests for septic bursitis: comparative study. BMJ 1998;316(7148):1877.

[11] Enzenauer RJ, Pluss JL. Septic olecranon bursitis in patients with chronic obstructive pulmonary disease. Am J Med 1996;100(4):479-80.

[12] Rubayi S, Montgomerie JZ. Septic ischial bursitis in patients with spinal cord injury. Paraplegia 1992;30(3):200-3.

[13] Drezner JA, Sennett BJ. Subacromial/subdeltoid septic bursitis associated with Isotretinoin therapy and corticosteroid injection. J Am Board Fam Pract 2004;17(4):299-302.

[14] Small LN, Ross JJ. Suppurative tenosynovitis and septic bursitis. Infect Dis Clin North Am 2005;19(5):991-1005.

[15] Dich VQ, Nelson JD, Haltalin KC. Osteomyelitis in infants and children: a review of 163 cases. Am J Dis Child 1975;129(11):1273-8. 